\title{
MECANISMOS DE EMPODERAMENTO: O QUE DIZEM OS AGENTES COMUNITÁRIOS DE SAÚDE (ACS) A RESPEITO DA SAÚDE SEXUAL NA ATENÇÃO BÁSICA
}

\author{
MECANISMOS DE EMPODERAMIENTO: LO QUE DICEN LOS AGENTES \\ COMUNITARIOS DE SALUD (ACS) AL RESPECTO DE LA SALUD SEXUAL EN \\ LA ATENCIÓN BÁSICA
}

\section{EMPOWERMENT MECHANISMS: WHAT THE COMMUNITY HEALTH AGENTS (CHA) SAY ABOUT SEXUAL HEALTH IN BASIC CARE}

\author{
Thiago Rubens da Silva ${ }^{1}$; Paulo Marcelo Freitas de Barros ${ }^{2}$
}

DOI: https://doi.org/10.31692/978-65-991061-9-4.14-28

\section{RESUMO}

Um dos maiores desafios dos tempos atuais está sendo o deixar de pensar e agir de forma fragmentada para o pensar e agir de forma sistêmica e interconexa. É comum o hábito de se pensar a sexualidade como sendo apenas o ato sexual, sendo essa, uma redução de algo muito mais amplo. O proposito desta pesquisa foi estudar a opinião dos Agentes Comunitários de Saúde (ACS) em relação ao nível de abrangência dos conceitos sobre saúde, sexualidade e solução de problemas vivenciados por quatro ACS que atuam em territórios assistidos pela Estratégia de Saúde da Família no Município de Olinda. Na análise qualitativa das respostas foram priorizados os seguintes itens: a compreensão de saúde com um direcionamento para os aspectos: físico, mental, espiritual, social e ambiental. Em seguida, a compreensão em relação à saúde sexual e a possibilidade de sua transmissão de saúde em relação aos cinco aspectos supracitados. Após a realização de quatro entrevistas semiestruturadas a análise de conteúdo revelou que o concito de saúde, apresentou características sistêmicas abordando as dimensões física, mental e social, não houve menções em relação aos aspectos espiritual e ambiental. Já no conceito de saúde sexual, houve um predomino de aspectos relacionados às doenças sexualmente transmissíveis, mas, também, ocorreu relatos dos aspecto mental e social. O tema transmissão de saúde sexual chegou a ser referido por dois dos entrevistados. $\mathrm{Na}$ área da sexualidade, os principais problemas referidos foram: iniciação precoce à vida sexual, resistência à prevenção, tabu sobre sexualidade, ausência de sexualidade na terceira idade e o preconceito sexual. Quanto à abrangência das soluções constatou-se que os entrevistados fizeram uma abordagem voltada apenas para os aspectos ligados à gestão e sócio-culturaleducacional. Em relação ao seminário, foram distribuídos um resumo dos resultados e a cartilha. Os participantes referiram não lidaram habitualmente com os conteúdos sobre saúde sistêmica, mas sim com apenas patologias. Além disso, relataram não lidarem com o aspecto espiritual no âmbito da atenção básica. De forma geral, constatou-se a necessidade de se aprofundar os temas supracitados tanto na formação profissional quanto em constantes capacitações da equipe de saúde na UBS.

Palavras-chave: Sexualidade, Saúde Pública, Centro de saúde.

\section{RESUMEN}

Uno de los mayores desafíos en los tiempos actuales es el dejar de pensar y actuar de forma fragmentada para pensar y actuar de forma sistémica e interconectada. Es común el hábito de pensar en la sexualidad como algo referido a apenas el acto sexual, siendo esa una reducción

\footnotetext{
${ }^{1}$ Fonoaudiologia, UNICAP, ichthiago@gmail.com

${ }^{2}$ Fonoaudiologia, UNICAP, paulofreitasb@gmail.com
} 
de algo que es mucho más amplio. El propósito de esta investigación fue estudiar la opinión de los Agentes Comunitarios de Salud (ACS) en relación al nivel de alcance de los conceptos sobre salud, sexualidad y la solución de problemas experimentados por cuatro ACS que trabajan en territorios asistidos por la Estrategia de Salud de la Familia en el Municipio de Olinda. En el análisis cualitativo de las respuestas fueron priorizados los siguientes aspectos: la comprensión de la salud con un direccionamiento para los aspectos: físico, mental, espiritual, social y ambiental. En seguida, la comprensión en relación a la salud sexual y la posibilidad de su transmisión de salud en relación a los cinco aspectos supracitados. Después de la realización de cuatro entrevistas semiestructuradas, el análisis de contenido reveló que el concepto de salud presentó características sistémicas abordando las dimensiones física, mental y social, no hubo menciones en relación a los aspectos espiritual y ambiental. En el concepto de salud sexual, hubo un predominio de aspectos relacionados a las enfermedades de transmisión sexual pero, también ocurrieron relatos de los aspectos mental y social. El tema transmisión de salud sexual llegó a ser mencionado por dos entrevistados. En el área de la sexualidad, los principales problemas mencionados fueron: inicio precoz en la vida sexual, resistencia a la prevención, tabú sobre sexualidad, ausencia de sexualidad en el adulto mayor y el preconcepto sexual. En cuanto al alcance de las soluciones, se constató que los entrevistados realizaron un abordaje enfocado apenas en los aspectos referidos a la gestión y la educación sociocultural. En relación al seminario, fueron distribuídos un resumen de los resultados y un folleto. Los participantes manifestaron que no lidiaron habitualmente con contenidos sobre salud sistémica, pero si apenas con patologías. Además, relataron que no trabajaron con el aspecto espiritual en el ámbito de la atención básica. De forma general, se constató la necesidad de profundizar en los temas supracitados tanto en la formación profesional como en las constantes capacitaciones del equipo de salud en la UBS.

Palabras-claves: Sexualidad, Salud Pública, Centro de Salud.

\section{ABSTRACT}

One of the biggest challenges of the present time is to move from thinking and acting in a fragmented way to thinking and acting in a systemic and interconnected way. It is common to think of sexuality as merely the sexual act, which is a reduction of something much broader. The purpose of this research was to study the opinion of the Community Health Agents (CHA) regarding the level of comprehension of the concepts on health, sexuality and problem solving experienced by four CHAs acting in territories assisted by the Family Health Strategy in the Municipality of Olinda. In the qualitative analysis of the answers, the following items were prioritized: the understanding of health with a direction to the aspects: physical, mental, spiritual, social and environmental. Then, the understanding of sexual health and the possibility of its transmission of health in relation to the five aspects mentioned above. After conducting four semi-structured interviews, the content analysis revealed that the health concern presented systemic characteristics addressing the physical, mental and social dimensions, there were no mentions regarding the spiritual and environmental aspects. In the concept of sexual health, there was a predominance of aspects related to sexually transmitted diseases, but there were also reports of mental and social aspects. The subject of sexual health transmission was mentioned by two of the interviewees. In the area of sexuality, the main problems mentioned were: early initiation into sexual life, resistance to prevention, taboo on sexuality, lack of sexuality in old age and sexual prejudice. Regarding the comprehensiveness of the solutions, it was found that the interviewees took an approach focused only on aspects related to management and socio-cultural-educational. Regarding the seminar, a summary of the results and the booklet were distributed. Participants reported that they did not usually deal with systemic health content, but only with pathologies. In addition, they reported not dealing with the spiritual aspect in primary care. In general, it was noted the need to deepen the 
aforementioned themes both in vocational training and in constant training of the health team at UBS.

Keywords: Sexuality, Public Health, Health Center.

\section{INTRODUÇÃO}

O presente estudo foi desenvolvido no contexto da Atenção Básica-AB enfocando a solução de problemas na área de transmissão de saúde e sexualidade. De acordo com a portaria 2.436 de 21 de setembro de 2017, que regula as normas da Assistência Básica no âmbito do SUS, a AB é o nível de atenção preferencial responsável pela entrada do usuário na Rede de Assistência à Saúde. Mundialmente, esse primeiro nível responsabiliza-se por solucionar aproximadamente $80 \%$ dos problemas.

Nesse contexto, é importante saber a opinião dos Agentes Comunitários de Saúde-ACS e como lidam com essa temática na $\mathrm{AB}$. Diante disso, é importante utilizarmos um conceito de saúde que abranja a complexidade de tais dimensões e inclua o tema, não menos complexo, da sexualidade que, particularmente, precisa ser visto muito além do ponto de vista da saúde como ausência de doenças. E necessário que a sexualidade na saúde seja percebido de um ponto de vista sistêmico envolvendo o corpo, a mente, a espiritualidade, a sociedade e o meio ambiente.

Para Barros (2003) a Soluciologia é a área do conhecimento que tem a solução como objeto de estudo que, numa visão sistêmica em saúde, é bem mais complexa que os problemas por envolver diversas áreas simultaneamente: gestão, finanças, legislações, múltiplas especialidades, diferentes níveis de comunicação, mudanças de comportamento, dentre outras.

O ACS tem um papel importante na $\mathrm{AB}$, segundo a portaria $\mathrm{N}^{\circ} 648$, DE 28 DE MARÇO DE 2006, define suas várias atribuições, que entre elas se destacam: desenvolver ações que busquem a integração entre a equipe de saúde e a população adscrita à UBS, articular a micro área colocando-a em contato permanente com as famílias, desenvolver ações educativas visando à promoção da saúde e a prevenção das doenças de acordo com o planejamento da equipe. O trabalho do ACS é considerado uma extensão dos serviços dentro das comunidades, já que é um membro da comunidade, o que ajuda a criar confiança e vínculo mutuamente estabelecidos entre eles.

Se a AB é considerada a porta de entrada nos Sistema Único de Saúde-SUS, o ACS pode ser considerado a "porta de entrada" do usuário na AB devido ao íntimo convívio desse profissional com os moradores de cada território e com as equipes de saúde. Valendo-se dessa proximidade, esse estudo justifica-se pela necessidade de fortalecimento das relações na $\mathrm{AB}$ a partir da sua missão de promoção de saúde e não apenas da premissa curativa de suas intervenções. 
Além disso, é muito precário o quantitativo de estudos que focalizem a "solução de problemas" na $\mathrm{AB}$ e, em especial, ao lidarem com a temática da transmissão de saúde no âmbito da sexualidade. Responsabilizando-se por ações resolutivas em saúde, a AB é um ambiente favorável à expansão de tais conceitos. Sore a solução de problemas, Barros (2003) refere que, devido à sua complexidade e importância, esse tema precisa existir contextualizado em todos os níveis de educação. A principal contribuição do estudo foi identificar, no cotidiano dos ACS, as principais lacunas a respeito da amplitude do conceito de saúde, resolução de problemas e transmissão de saúde na área da sexualidade. Certamente, ele pode servir de modelo para que seja aperfeiçoado, adaptado e replicado para as necessidades epidemiológicas de outros municípios.

Devido a atual capacitação oferecida aos ACS muito provavelmente, há predomínio do conceito fragmentado de saúde, pouca abrangência na solução de problemas e pouco conhecimento sobre transmissão de saúde na área de sexualidade. Portanto, o objetivo do estudo foi identificar a opinião dos ACS a respeito dos temas em questão além disso, foi realizado um seminário temático na comunidade envolvendo os ACS para que tais conteúdos pudessem ser melhor aprofundados no seu cotidiano.

\section{FUNDAMENTAÇÃO TEÓRICA}

Um dos maiores desafios dos tempos atuais está sendo o deixar de pensar e agir de forma fragmentada para o pensar e agir de forma sistêmica e interconexa. É comum o hábito de se pensar a sexualidade como sendo apenas o ato sexual, sendo essa, uma redução de algo muito mais amplo (PINTO, 2002). De acordo com o Ministério da Saúde (2006), a sexualidade é muito mais do que sexo, é um aspecto central da vida das pessoas e envolve sexo, papéis sexuais, orientação sexual, erotismo, prazer, envolvimento emocional, amor e reprodução.

Epistemologicamente, a compreensão da ciência sobre a realidade que nos cerca, tem sofrido diversas transições. Uma das mais significativas está sendo o mirarmos de uma percepção newtoniana cartesiana fragmentada (linear) em que o mundo é constituído de partes isoladas e independentes, para uma percepção sistêmica (Não linear) em que o mundo é percebido como sendo uma complexa rede interativa de infinitas relações (CAPRA, 2002).

O autor ainda revela, o fato de não percebermos a realidade como algo único e unificado, fragmentando o mundo e ignorando a interligação dinâmica de todas as coisas, constitui-se no erro de percepção responsável por nossos problemas, na ciência, em nossa vida pessoal, em nossa sociedade e no meio ambiente. A partir dessa perspectiva sistêmica, como revisitar nossos conceitos de saúde e sexualidade no contexto da atenção básica? Além do estudo das patologias, 
seria interessante estudarmos a possibilidade de se transmitir saúde? Usualmente, a transmissão de saúde é compreendia apenas com a transmissão de informações para se evitar doenças (CARVALHO,1999).

Além disso faz se necessário utilizarmos um conceito de saúde que abranja a complexidade de tais dimensões e inclua o tema, não menos complexo, da sexualidade que, particularmente, precisa ser visto muito além do ponto de vista da saúde como ausência de doenças, é necessário vermos a sexualidade na saúde do ponto de vista sistêmico que inclua o físico, o psicológico, o espiritual, o social e o ambiental (CAPRA, 2002).

Inevitavelmente, o conceito de saúde e o estabelecimento de práticas dependem do grau de conhecimento disponível na ciência e do grau de conhecimento particular de cada profissional de saúde (FONSECA, 2007).

Concordando com Carvalho (1999) é realmente importante estar bem informado tanto para se ter saúde quanto para resolver os problemas de saúde. Uma vez que o conceito ampliado de saúde e, também, de sexualidade envolvem todos os aspectos de vida do ser humano, a convivência de quem não tem saúde com quem a possui, por ressonância, também é fundamental como forma de aprendizagem. Nos sistemas vivos, segundo Capra (2002) a bioressonância pode ser observada em padrões de comportamento ou na aquisição e uso da linguagem. Silva Santos (2016) também confirma esse padrão de imitação comportamental, por ressonância, em nossos hábitos sociais e de consumo. Portanto, quanto mais sadia uma pessoa ou uma sociedade estiver, mais fácil será a transmissão de saúde.

Para Barros (2003) a soluciologia é a área do conhecimento que tem a solução como objeto de estudo que, numa visão sistêmica em saúde, são bem mais complexas que os problemas por envolverem diversas áreas simultaneamente: gestão, finanças, legislações, múltiplas especialidades, diferentes níveis de comunicação, mudanças de comportamento, dentre outras.

No estudo de uma solução pode-se optar por dois caminhos básicos: 1) verificar a abrangência da solução ou 2) verificar se a solução proposta teria condições de resolver o problema ou não. No presente estudo optou-se pela primeira possibilidade, ou seja, o estudo da abrangência identificando, em quantas e quais áreas as soluções foram relatadas e qual o nível de complexidade desse relacionamento (de superficial até muito complexo) em seis áreas básicas: gestão, jurídico, formação de redes, mudanças de comportamento, mudanças socioculturais- educacionais e aspectos financeiros BARROS (2013).

A problemática sexual, muitas vezes extrapola o aspecto físico da disfunção, originando problemas na vida profissional, nos relacionamentos familiares e sociais, na relação com seu 
parceiro e em sua saúde psíquica (FEIJÓ, 2007).

Durante a confecção do presente estudo, ao realizarmos um levantamento feito nas bases de dados BVS, LILACS e SCIELO, percebeu-se uma escassez de estudos que abordem a sexualidade e a transmissão de saúde numa visão sistêmica. É natural que tenhamos dificuldades em lidarmos com a sexualidade sob a nova concepção de saúde do ponto de vista sistêmico envolvendo os cinco aspectos constituintes do conceito de saúde mencionado anteriormente.

No Brasil, a Estratégia de Saúde da Família (ESF) representa o primeiro nível de atenção que considera os indivíduos, famílias e comunidades como objetos centrais em ações de prevenção, promoção e tratamento (FIALHO, 2016). Para isso, conta com uma equipe multiprofissional responsável pela saúde da população local e uma de suas exigências é a presença de um ACS. Lotado geralmente em Unidades Básicas de Saúde-UBS, este profissional executa atividades domiciliares, tais como visitas e orientações às famílias (COTTA, R. M. M., 2013).

O fato é que, os ACS são profissionais fundamentais para o funcionamento da promoção de saúde e o funcionamento da Atenção Básica. De acordo com a Portaria 2.439 de 21 de setembro de 2017, a Atenção Básica caracteriza-se por um conjunto de ações de saúde, no âmbito individual e coletivo, que abrange a promoção, a proteção da saúde, com o objetivo de desenvolver uma atenção integral que impacte na situação de saúde, autonomia das pessoas, nos determinantes e condicionantes de saúde das coletividades (BRASIL, 2017).

Dessa forma fez se necessário saber como os ACS estão lidando com a transição do conceito de saúde em sua aplicabilidade na área da sexualidade para que, de forma interconexa, possamos ter cada vez mais saúde do ponto de vista sistêmico integrando, apropriadamente, o corpo, a mente, a espiritualidade, a sociedade e o meio ambiente.

\section{METODOLOGIA}

\section{Coleta e Análises dos Dados:}

A presente pesquisa foi uma continuidade do projeto aprovado pelo Comitê de Ética da Unicap-parecer:025/2016 (CAAE: 56350316.7.0000.5206) e realizada entre os meses de agosto de 2018 até junho de 2019 no Programa de Bolsas de Iniciação Científica Universidade Católica de Pernambuco-UNICAP. O estudo estará dividido em duas etapas. Uma voltada à coleta de dados e outra voltada para a realização de um seminário.

Na primeira etapa, em relação à seleção dos Agentes Comunitários de Saúde, tivemos quatro indivíduos. Todos eles integrantes de Unidades Básicas de Saúde do Município de 
Olinda. Naturalmente, foram excluídos os que se recusaram a participar ou que estiveram ausentes no período por motivos alheios à pesquisa.

Após um estudo piloto para aprimoramento do instrumento de coleta, todos os participantes assinaram o TCLE (anexo I), responderam, a uma entrevista composta por quatro perguntas, realizadas pelos pesquisadores e gravadas por meio de um gravador de voz em ambiente reservado (anexo II). A análise de conteúdo das respostas forma orientada por alguns princípios norteadores inspirados no modelo proposto por Laurence Bardin (2011) e por princípios da soluciologia propostos por Barros (2018) (Anexo III). Os dados foram coletados através de entrevista piloto realizada com um profissional de saúde.

A análise de conteúdo das respostas ocorreu a partir do seguinte roteiro: foi realizada uma leitura minuciosa da transcrição, a fim de formular uma ideia geral do que foi abordado. Em seguida, identificadas as unidades significativas de interesse para depois, organizá-las e transforma-las em categorias que correspondam ao agrupamento de unidades de significado, buscando traduzir a essência da fala do entrevistado em relação aos objetivos do estudo. Na análise qualitativa das respostas foram priorizados os seguintes itens: a compreensão de saúde que o entrevistado relatou com um direcionamento para os aspectos: físico, mental, espiritual, social e ambiental. Em seguida, a compreensão do entrevistado em relação à saúde sexual e a possibilidade de sua transmissão em relação aos cinco aspectos supracitados. De forma complementar, foram investigados os principais problemas e as principais soluções, na área da sexualidade, vivenciados no cotidiano da respectiva UBS. Em ambos os casos, a análise se conteúdo teve como foco, apenas o nível de abrangência das respostas e não a viabilidade ou não de seus conteúdos.

Na segunda etapa, foi realizada uma oficina com todos os seguimentos participantes com o intuito de apresentar os resultados do estudo e elaborar, conjuntamente, uma cartilha instrucional sobre o tema saúde sexualmente transmissível dentro do conceito de saúde sistêmica.

\section{RESULTADOS E DISCUSSÃO}

Os resultados serão apresentados de forma textual em relação os quatros Agentes Comunitários de Saúde entrevistadas, sendo ACS1 a primeira, ACS2 a segunda, ACS3 a terceira e ACS4 a quarta. Inicialmente os entrevistados foram questionados no que diz respeito o conceito de saúde do ponto de vista sistêmico. 


\section{Conceito de saúde sistêmico}

Quadro 1: distribuição do espectro de abrangência do conceito geral de saúde.

\begin{tabular}{|c|c|c|c|c|c|}
\hline ACS & Física & Mental & Espiritual & Social & Ambiental \\
\hline 1 & $\mathrm{x}$ & $\mathrm{x}$ & & $\mathrm{x}$ & \\
\hline 2 & $\mathrm{x}$ & & & $\mathrm{x}$ & \\
\hline 3 & $\mathrm{x}$ & $\mathrm{x}$ & & $\mathrm{x}$ & \\
\hline 4 & $\mathrm{x}$ & & & $\mathrm{x}$ & \\
\hline
\end{tabular}

A seguir serão apresentados recortes das opiniões relatas pelos os ACS sobre as suas concepções em saúde englobando ou não os cinco aspectos investigados (físico, mental, espiritual, social e ambiental:

- $\quad$ ACS1 = “É ver o paciente como um todo, não só a causa médica. Porque existe vários problemas vários, familiares como, problema com o filho, das drogas, problema com marido, problema com doença e é preciso ver essa parte também”. Nas respostas da entrevistada observou-se apenas a presença dos aspectos físico, mental e social dento do conceito de saúde referido.

- $\quad \mathbf{A C S 2}=$ "Para ter saúde tem que ter um emprego, ter salário digno... Uma pessoa para ter saúde tem que ter lazer, tem que estar preparado fisicamente e financeiramente. Viver com dignidade. Se você tiver tudo isso irar ter mais saúde". Nas respostas da entrevistada observou-se apenas a presença dos aspectos físico e social dento do conceito de saúde referido.

- $\quad \mathbf{A C S 3}=$ "Uma pessoa saudável que não tenha nenhum problema de doença, tanto como mentalmente, intelectualmente que ela usufrua da sua vida com todo gozo". Nas respostas da entrevistada observou-se apenas a presença dos aspectos físico, mental e social dento do conceito de saúde referido.

- $\quad \mathbf{A C S 4}=$ "A integração do sistema político de saúde, cultural, econômico, porque tudo isso vai ajudar para uma saúde de qualidade". Nas respostas da entrevistada observouse apenas a presença dos aspectos físico e social dento do conceito de saúde referido.

$\mathrm{Na}$ "concepção de saúde" referida pelas entrevistadas em relação aos paradigmas sistêmico ou fragmentado, percebeu-se que os participantes apresentaram uma visão sistema em saúde alinhado com os preceitos da OMS (2006) caracterizarem "saúde" não apenas como a ausência de doença, mas como uma situação de bem-estar físico, mental e social. Entretanto, ao considerarmos os cinco aspectos juntos: físico, mental, espiritual, social e ambiental, 
perceberemos que nenhuma das entrevistadas abordou todos em seus discursos. Apesar da presença da visão sistêmica identificada, ela não totalmente abrangente. Houve predomínio dos aspectos físico e social e omissão dos aspectos espiritual e ambiental.

Nessa perspectiva utilizando o conceito de saúde no ponto de vista sistêmico, em relação ao conceito de saúde sexual os entrevistados foram questionados qual seria a sua opinião sobre o tema.

\section{Saúde sexual}

Quadro 2: distribuição do espectro de abrangência do conceito de saúde sexual.

\begin{tabular}{|c|c|c|c|c|c|}
\hline Sujeitos & Física & Mental & Espiritual & Social & Ambiental \\
\hline 1 & & $\mathrm{x}$ & & & \\
\hline 2 & $\mathrm{x}$ & $\mathrm{x}$ & & $\mathrm{x}$ & \\
\hline 3 & $\mathrm{x}$ & $\mathrm{x}$ & & $\mathrm{x}$ & \\
\hline 4 & $\mathrm{x}$ & $\mathrm{x}$ & & & \\
\hline
\end{tabular}

A seguir serão apresentados recortes das opiniões relatas pelos os ACS sobre o tema abordado acima.

- CSA1 = “É quando você está bem com você mesmo. Porque quando a pessoa não está bem psicologicamente não consegue ter uma relação com o marido”.

- $\quad \mathbf{A C S 2}=$ "Se você tem a base que é ter um emprego, lazer, estar preparado fisicamente e financeiramente. Isso tudo contribui para que você sinta mais prazer sexualmente. Mas se você estiver mal e não tiver nada disso... Só o sexo. Você “esfria' também. E se você não tiver bem fisicamente não vai ter ânimo para a sexualidade vai faltar o prazer e também só a cama não serve meu filho tem que ter os outros prazeres".

- $\quad \mathbf{A C S 3}=$ "Tem tabus né... Porque a gente para ter uma saúde sexual tem que está bem de mente, tem que está bem com todos, tem que está bem com o parceiro. O parceiro satisfazer a gente nos pontos que a gente precisa que.... Hoje tem muito tabu muitas mulheres não conseguem ter orgasmos e... Acho que falta hoje muita parceria, contato”.

- ACS4= “ Saúde sexual... É quando a pessoa é bem resolvida, porque a saúde sexual é bom para o corpo e até para cabeça. É necessário para que se tenha uma saúde de qualidade, ter sexo... Porque faz parte da vida"

Em relação ao tema "saúde sexual", ficou evidente que dentre os quatros participantes, todos relataram uma abordagem focada nas dimensões física, mental e social. Segundo a ACS A1 a sexualidade valoriza a sua relação com ela mesma. Além disso, a saúde sexual seria uma 
interação entre os aspectos físico, social e mental que serão determinantes para uma vida sexual prazerosa visão percebida pela ACS A2. Para ter uma saúde sexual é preciso está com uma mente sadia, está bem com o outro e com o mundo; socialmente existem tabu sobre a temática que por esse fato muitas mulheres não conseguem sentir prazer sexual e ter seus orgasmos, segundo relato da ACS A3. Dessa forma, a sexualidade faz parte da vida e é necessário para que se tenha uma saúde de qualidade, segundo a ACS A4.

Quanto a abrangência do conceito de sexualidade de saúde sexual ficou evidente o predomínio das dimensões física, mental e social com omissão das dimensões espiritual e ambiental. Utilizando o conceito de saúde referido no estudo os participantes foram questionados sobre transmissão de saúde sexual.

\section{Saúde sexualmente transmissível}

Quadro 3: distribuição do espectro de abrangência para a possibilidade de a saúde ser transmissível.

\begin{tabular}{|c|c|c|c|c|c|}
\hline Sujeitos & Física & Mental & Espiritual & Social & Ambiental \\
\hline 1 & $\mathrm{x}$ & $\mathrm{x}$ & & & \\
\hline 2 & $\mathrm{x}$ & & & & \\
\hline 3 & $\mathrm{x}$ & & & & \\
\hline 4 & $\mathrm{x}$ & $\mathrm{x}$ & & & \\
\hline
\end{tabular}

A seguir serão apresentados recortes das opiniões relatas pelos os ACS sobre o tema abordado acima:

- $\quad \mathbf{A C S 1}=$ "Se a pessoa tiver uma determinada doença e passa para a esposa, ela desabafa com uma amiga intima, se essa amiga estiver bem de saúde irar transmitir um conforte para aquela dificuldade que ela estar passando, explicando para ela o que é preciso fazer, isso irar transmitir autoestima para ela".

- $\quad \mathbf{A C S 2}=$ " Se você se previne e tem os seus cuidados com o seu corpo, assim você vai ter saúde e vai oferecer uma vida conjugal ao seu parceiro sadia. Assim você vai oferecer segurança ao seu parceiro"

- $\mathbf{A C S 3}=$ "Se você está todo sadio, você tem uma relação prazerosa, aquilo está transmitindo saúde, porque faz bem para o corpo, faz bem para pele"

- $\mathbf{A C S 4}=$ “ Com prevenção, usando camisinha... E também dialogando com o parceiro, mostrando a necessidade da prevenção e nessa conversa saber o que é bom para ele, como para os dois... Não forçar o parceiro a ter uma relação que não quer. Porque na minha área converso muito sobre isso e geralmente as reclamações é que eles só pensam nele, não querem saber se a parceira está satisfeita, se ela está gostando. 
Em relação à transmissão de 'saúde sexual' ficou evidente dentre os quatros participantes, dois ACS1 E ACS2 privilegiaram uma abordagem focada na dimensão física a partir da prevenção de doenças sexualmente transmissíveis. Entretanto, foi relatada a possibilidade da transmissão de autoestima e segurança, dois aspectos mentais. ACS3 E ACS4 abordaram à transmissão de saúde sexual como, ter uma relação sexual prazerosa, satisfazendo o parceiro sexualmente, o autocuidado, diálogo e respeito.

De forma geral, apesar de relatos pouco abrangentes sobre a possibilidade de se transmitir saúde através da sexualidade, ficou evidente que tal conteúdo foi um tema pouco discutido no âmbito da Atenção Básica que, no Brasil, de acordo com a Portaria 2.436, ela é a porta de entrada do sistema de saúde e, dentre outras, tem a função de promover, prevenir, diagnosticar e tratar os problemas de saúde. A atenção básica é, portanto, um local propício para que seja aprofundado os conhecimentos sobre saúde e não apenas sobre doenças.

\section{Problemas e Soluções}

Primeiramente, serão apresentados os problemas coletados. Depois, as soluções e, ao final, as análises a partir dos referenciais de abrangência adotado pelo autor BARROS (2013).

Vale ressaltar que não foi objetivo do estudo verificar se as soluções teriam condições de resolver o problema ou não. Optou-se por verificar apenas a abrangência das soluções que foram referidas identificando-se em quantas e quais áreas as soluções foram relacionadas aos aspectos: gestão, jurídico, formação de redes, mudanças de comportamento, mudanças socioculturais- educacionais e aspectos financeiros. Em seguida, será apresentada a nota/conceito atribuída a cada solução.

\section{ACS 1}

\section{PROBLEMAS:}

1- Prevenção; 2 - Tabu sobre sexualidade; 3- Falta de informação na primeira relação sexual (Educação sexual); 4 Orientação familiar; 5- Sexualidade na terceira idade.

\section{SOLUÇÕES:}

1-Convencer as pessoas a usar preservativo para prevenir as doenças; 2- Elaboração de palestras sobre a temática; 3- Elaboração de palestras sobre a temática; 4-: os profissionais de saúde orientar aos pais conversarem com os filhos sobre sexualidade. 5- Conscientizar aos idosos que eles podem ter uma vida sexual. 
Quadro 1: distribuição do espectro de abrangência para as soluções (S1).

\begin{tabular}{|c|c|c|c|c|c|c|c|c|c|c|c|}
\hline \multirow{3}{*}{ Sujeito } & \multicolumn{6}{|c|}{ Aspectos } & \multirow{3}{*}{ Nota } & \multicolumn{4}{|c|}{ Conceito } \\
\hline & Gestão & Redes & Sócio/c/e & Mudança/Cpto. & Financeir & Jurid. & & $\mathrm{r}$ & $\mathrm{R}$ & B & $\mathrm{E}$ \\
\hline & & & & & & & & & & & \\
\hline 1 & 2 & 0 & 1 & 0 & 0 & 0 & 3 & $\mathrm{X}$ & & & \\
\hline
\end{tabular}

r-ruim (0 a 3 pontos); R-regular (4 a 11); B-bom (12 a 14); E-excelente (16 a 18)

\section{ACS 2}

\section{PROBLEMAS:}

1- Sexualidade precoce; 2 - Prevenção; 3- Prostituição; 4 Falar sobre sexualidade na terceira idade; 5- Falta de informação.

\section{SOLUÇÕES:}

1- Elaboração de palestras sobre DST; 2- Elaboração de palestras sobre DST; 3- Incentivo educativo ao uso de preservativo; 4-: "se precisar abordar o assunto, mas é normal quando se chega uma certa idade parar a vida sexual'. 5- Um trabalho informativo sobre sexualidade para as pessoas.

Quadro 2: distribuição do espectro de abrangência para as soluções (S2).

\begin{tabular}{|c|c|c|c|c|c|c|c|c|c|c|c|}
\hline \multirow[b]{2}{*}{ Sujeito } & \multicolumn{6}{|c|}{ Aspectos } & \multirow{2}{*}{ Nota } & \multicolumn{4}{|c|}{ Conceito } \\
\hline & Gestão & Redes & Sócio/c/e & Mudança/Cpto. & Financeiro & Jurid & & $\mathrm{r}$ & $\mathrm{R}$ & B & $\mathrm{E}$ \\
\hline 2 & 2 & 0 & 1 & 0 & 0 & 0 & 3 & $\mathrm{x}$ & & & \\
\hline
\end{tabular}

r-ruim (0 a 3 pontos); R-regular (4 a 11); B-bom (12 a 14); E-excelente (16 a 18)

\section{ACS 3}

\section{PROBLEMAS:}

1- Tabu em falar sobre sexualidade; 2 - Educação sexual; 3- Prazer sexual na relação; 4 Autoestima feminina é necessário para satisfazer na relação; 5- Preconceito sexual.

\section{SOLUÇÕES:}

1- Orientação, elaboração de palestras sobre sexualidade ; 2- Orientação, elaboração de palestras sobre a temática; 3- Orientação com os parceiros das mulheres e instigar as mulheres a conversar com os seus parceiros.; 4-: Que as mulheres recebam o apoio dos profissionais de saúde e também tenha um apoio psicológico; 5- Orientação, elaboração de palestras sobre a temática.

Quadro 3: distribuição do espectro de abrangência para as soluções (S3).

\begin{tabular}{|c|c|c|c|c|c|c|c|c|c|c|c|}
\hline \multirow[b]{2}{*}{ Sujeito } & \multicolumn{6}{|c|}{ Aspectos } & \multirow{2}{*}{ Nota } & \multicolumn{4}{|c|}{ Conceito } \\
\hline & Gestão & Redes & Sócio/c/e & Mudança/Cpto. & Financeiro & Jurid & & $\mathrm{r}$ & $\mathrm{R}$ & B & $\mathrm{E}$ \\
\hline 3 & 2 & 0 & 1 & 0 & 0 & 0 & 3 & $\mathrm{x}$ & & & \\
\hline
\end{tabular}

r-ruim (0 a 3 pontos); R-regular (4 a 11); B-bom (12 a 14); E-excelente (16 a 18) 


\section{$\operatorname{ACS} 4$}

\section{PROBLEMAS:}

1- Resistencia da prevenção; 2 - Iniciação precoce a vida sexual; 3- Prazer sexual na relação; 4 Tabu sexualidade na terceira idade; 5- Preconceito, mente fechada.

\section{SOLUÇÕES:}

1- Informação, elaboração de uma reciclagem que envolvesse os profissionais e a comunidade;

2- Elaboração de palestras sobre o assunto; 3 Trabalho educativo com elaboração de palestras que chamasse a comunidade, tanto as mulheres como os homens também; 4 Conversar com os idosos, incentivando e orientando que eles podem ter uma vida sexual; 5- Trabalhar o preconceito com informação para essas pessoas.

Quadro 4: distribuição do espectro de abrangência para as soluções (S4).

\begin{tabular}{|c|c|c|c|c|c|c|c|c|c|c|c|}
\hline \multirow{3}{*}{ Sujeito } & \multicolumn{5}{|c|}{ Aspectos } & \multicolumn{3}{|c|}{ Conceito } \\
\cline { 2 - 14 } & Gestão & Redes & Sócio/c/e & Mudança/Cpto. & Financeiro & Jurid & & r & R & B & E \\
\hline 1 & 2 & 0 & 1 & 0 & 0 & 0 & $\mathrm{x}$ & 3 & & & \\
\hline
\end{tabular}

r-ruim (0 a 3 pontos); R-regular (4 a 11); B-bom (12 a 14); E-excelente (16 a 18)

Ao observar todas as respostas pode-se constatar que todos os quatro entrevistados fizeram uma abordagem voltada apenas para os aspectos ligados à gestão e sócio-culturaleducacional, não foram abordados os itens jurídicos, parcerias, mudanças de comportamento e custos e nenhum entrevistado incluiu-se nas soluções. Desse modo o nível de abrangência foi classificado como ruim com três pontos.

\section{Seminário}

Ao final realizou-se um seminário para informar aos participantes da pesquisa os resultados obtidos. No momento da reunião, que constou de seis participantes e quatro pesquisadores, optou-se por uma roda de conversa. Primeiramente, foi distribuído um resumo dos resultados e a cartilha sobre saúde sexualmente transmissível. Durante a reunião os participantes referiram não lidaram habitualmente com os conteúdos sobre saúde, mas sobre patologias. Além disso, relataram não lidaram habitualmente com os conteúdos sobre saúde sistêmica, mas sim, com patologias. Foi necessário ampliar alguns conceitos como: transmissão de saúde, sexualidade e ressonância comportamental. Eles fizeram relatos de diversas experiências vividas na própria UBS. Relataram que a temática do estudo era muito interessante, como também, o fato pensar em saúde sem estar atrelado a doenças demostrarando interesse em buscar esse conhecimento. 


\section{CONSIDERAÇÕES FINAIS}

A partir do objetivo do estudo que foi estudar a opinião dos Agentes Comunitários de Saúde (ACS) a respeito das relações entre sexualidade e transmissão de saúde. Na análise do conteúdo percebeu-se que o conceito de saúde, identificado no discurso dos entrevistados, apresentou características sistêmicas abordando as dimensões física, mental e social, não houveram menções, por nenhum dos entrevistados, em relação aos aspectos espiritual e ambiental. Já no conceito de saúde sexual, houve um predomino de aspectos relacionados às doenças sexualmente transmissíveis, mas, também, ocorreu relatos dos aspecto mental e social. O tema transmissão de saúde sexual chegou a ser referido por dois dos entrevistados. A possibilidade de se transmitir saúde através da sexualidade foi um tema pouco discutido no âmbito da Atenção Básica. Na área da sexualidade, os principais problemas referidos na UBS, foram eles: iniciação precoce a vida sexual, resistência a prevenção, tabu sobre sexualidade, ausência de sexualidade na terceira idade e o preconceito sexual. Quanto à abrangência das soluções constatou-se que os entrevistados fizeram uma abordagem voltada apenas para os aspectos ligados à gestão e sócio-cultural-educacional, foram abordados os itens jurídicos, parcerias, mudanças de comportamento e custos. Desse modo o nível de abrangência foi classificado como ruim com três pontos. De forma geral, constatou-se a necessidade de se aprofundar os temas supracitados tanto na formação profissional quanto em constantes capacitações da equipe de saúde, particularmente, no âmbito da atenção básica que é a porta de entrada do sistema de saúde e, dentre outras, tem a função de promover, prevenir, diagnosticar e tratar os problemas de saúde.

\section{REFERÊNCIAS}

BARDIN, Laurence. Análise de conteúdo. São Paulo: Edições 70, 2011, 229

BARROS, P.M.F. A solução como objeto de estudo: Soluciologia. Olinda: Livro Rápido Elógica, 2013.

BARROS, M.F.B. Soluciologia: coleta de dados. Disponível em: <http://soluciologia.blogspot.com/>. Acesso em junho de 2019.

BRASIL. Ministério da Saúde. Portaria 2.436 de 21 de setembro de 2017. Brasília: Diário Oficial [da] República Federativa do Brasil, 2017.

CAPRA, Fritjof. Conexões ocultas: Ciência para uma vida sustentável. São Paulo. Cultrix, 2002. 296 p. Tradução de: The web of life; a new science understanding of living systems.

CARVALHO Teixeira, J. A. Psicologia da saúde e prevenção do cancro. Desafios para a 
psicologia da saúde. Análise Psicológica, 14 (1), 135-139. 1999.

CORDIOLI, S. Enfoque participativo no trabalho com grupos. In: BROSER, M. Metodologia participativa: uma introdução a 29 instrumentos. Porto Alegre: Tomo Editorial, 2001.

FEIJÓ, M. R. Práticas sistêmicas com casais e famílias com dificuldades afetivo-sexuais. (Orgs.), Sexualidade na família. (pp. 111-124). São Paulo: Expressão e Arte. 2007

FIALHO, Juliana Aparecida. Percepção dos Agentes Comunitários de Saúde sobre seu trabalho na estratégia de saúde da família. Minas Gerais: UF Viçosa, 2016.

FONSECA, Angélica Ferreira (Org.) O território e o processo saúde-doença. Rio de Janeiro: EPSJV/Fiocruz, 2007.

Ministério da Saúde. Direitos sexuais, direitos reprodutivos e métodos anticoncepcionais. Secretaria de Atenção à Saúde, Departamento de Ações Programáticas Estratégicas. Brasília DF. Disponível em: $<$ http://docs.google.com/viewer?a=v\&q=cache:pmEmCS7X8b4J:portal.saude.gov.br/portal/ar quivos/pdf/cartilha_direitos_sexuais_2006.pdf>. Acesso em 30 jan. 2018.

PINTO, E. B. Sexualidade: um bate-papo com o psicólogo. São Paulo: Paulinas, 2002.

RIBEIRO, J. P., \& SANTOS, A. (2005). Estudo exploratório da relação entre função eréctil, disfunção eréctil e qualidade de vida em homens portugueses saudáveis. Análise Psicológica, 3(23), 341-349.

ROLIM, L. B. Saúde em Debate. Rio de Janeiro, v. 37, n. 96, p. 139-147, jan./mar. 2013. 